\title{
Influence of Si doping on InAs/GaAs quantum dot solar cells with AIAs cap layers
}

Dongyoung Kim

Mingchu Tang

Jiang Wu

Sabina Hatch

Yurii Maidaniuk

Vitaliy Dorogan

Yuriy I. Mazur

Gregory J. Salamo

Huiyun Liu 


\title{
Influence of Si doping on InAs/GaAs quantum dot solar cells with AlAs cap layers
}

\author{
Dongyoung Kim ${ }^{* a}$, Mingchu Tang ${ }^{\text {a }}$, Jiang Wu ${ }^{\text {a }}$, Sabina Hatch ${ }^{\text {a }}$, Yurii Maidaniuk ${ }^{\text {b }}$, Vitaliy \\ Dorogan $^{b}$, Yuriy I. Mazur ${ }^{b}$, Gregory J. Salamo ${ }^{b}$, Huiyun Liu ${ }^{\text {a }}$ \\ ${ }^{a}$ Department of Electronic and Electrical Engineering, University College London, Torrington \\ Place, London WC1E 7JE, United Kingdom; ${ }^{\mathrm{b}}$ Institute for Nanoscience and Engineering, University \\ of Arkansas, Fayetteville, Arkansas 72701, United States of America \\ ${ }^{*}$ Email: d.kim@ucl.ac.uk
}

\begin{abstract}
In this work, the effect of Si doping on InAs/GaAs quantum dot solar cells with AlAs cap layers is studied. The AlAs cap layers suppress the formation of the wetting layer during quantum dot growth. This helps achieve quantum dot state filling, which is one of the requirements for strong sub-bandgap photon absorption in the quantum dot intermediate band solar cell, at lower Si doping density. Furthermore, the passivation of defect states in the quantum dots with moderate $\mathrm{Si}$ doping is demonstrated, which leads to an enhancement of the carrier lifetime in the quantum dots, and hence the opencircuit voltage.
\end{abstract}

Keywords: Quantum dot solar cells, intermediate band solar cells, molecular beam epitaxy

\section{INTRODUCTION}

Since the concept of the intermediate band solar cell (IBSC) was proposed in 1997, significant efforts have been made to realise IBSCs with efficiencies that exceed the Shockley-Queisser limit of $31 \%{ }^{1}$. The IBSC utilises a collection of intermediate levels within the bandgap, called the intermediate band (IB), to absorb sub-bandgap energy photons. Its potential to exceed the Shockley-Queisser limit and reach the theoretical limit of $63.2 \%{ }^{2}$ arises from the additional photocurrent generated by the sub-bandgap photon absorption. One of the candidates for the high-efficiency IBSC is the quantum dot intermediate band solar cell (QD-IBSC), which utilises the discrete nature of the carrier density of states and the bandgap tunability of quantum dots (QDs). In a QD-IBSC, the IB is formed by the confined electron states in the QD array.

However, there have been several challenges in implementing high-efficiency QD-IBSCs. Firstly, thermal decoupling needs to be achieved between the IB and the conduction band (CB) of wetting layer (WL). The thermal coupling between the IB and $\mathrm{CB}$ enables the carriers to be thermally excited or relaxed between the IB and the $\mathrm{CB}^{3-5}$. This thermal process leads to suppression of the second-photon absorption, and hence a lower open-circuit voltage $\left(\mathrm{V}_{\mathrm{OC}}\right)^{6,7}$. Tutu et al. have demonstrated suppression of thermal escape of electrons in QDs by removing the WL ${ }^{8}$. Lam et al. have also reported reduction of thermal coupling of QD states from the WL by introducing a potential barrier between the QDs and WL via Si doping ${ }^{9}$. Secondly, the accumulated strain from the QDs leads to the formation of threading dislocations ${ }^{10-12}$. The strain-induced dislocations of the QDs result in short minority carrier lifetime, and cause difficulties in increasing the number of the QD layers that are needed to maximise the photon absorption by QDs ${ }^{11-14}$. Furthermore, the performance of IBSCs relies on an IB that is partially filled with electrons ${ }^{15,16}$. For strong sub-bandgap photon absorption, the IB needs to have empty states to receive the electrons pumped from the VB, and states filled with electrons to pump electrons to the $\mathrm{CB}$. Luque et al. proposed the use of doping as a method to achieve partial filling of the confined states in the IB ${ }^{17}$.

Physics, Simulation, and Photonic Engineering of Photovoltaic Devices VI, edited by Alexandre Freundlich, Laurent Lombez, Masakazu Sugiyama, Proc. of SPIE Vol. 10099, 100990H · C 2017 SPIE CCC code: $0277-786 \mathrm{X} / 17 / \$ 18 \cdot$ doi: $10.1117 / 12.2250328$ 
In this work, we report the saturation of strain-induced dislocations and QD state filling by Si-doping QDs in InAs/GaAs QDSCs with AlAs CLs. Previously, we demonstrated that the deposition of AlAs CLs on InAs QDs could suppress the formation of the $\mathrm{WL}^{8}$. Consequently, the effective bandgap of the QDSC was increased, which in turn led to the increase in the thermal activation energy and the $\mathrm{V}_{\mathrm{OC}}$. In this study, in addition to the enhancement of the $\mathrm{V}_{\mathrm{OC}}$ by applying AlAs CLs, a further increase in the $\mathrm{V}_{\mathrm{OC}}$ by $\sim 44 \mathrm{mV}$ is observed by passivating the defect states with moderate Si doping (6 e/dot). In addition, QD state filling, which is essential for strong two-photon absorption, is observed with significantly lower Si doping densities when compared with that of our previous study ${ }^{9}$.

\section{GROWTH DETAILS}

The InAs/GaAs QDSC samples with AlAs CLs were grown by a solid-source molecular beam epitaxy (MBE) on $\mathrm{n}^{+}$ GaAs (100) substrates. All SCs were grown with the same p-i-n structure that consists of a $200 \mathrm{~nm} \mathrm{n}^{+}$GaAs buffer layer, $30 \mathrm{~nm} \mathrm{n}^{+} \mathrm{Al}_{0.35} \mathrm{Ga}_{0.65} \mathrm{As}$ back surface field, $1000 \mathrm{~nm} \mathrm{n}{ }^{-}$GaAs base, $250 \mathrm{~nm} \mathrm{p} \mathrm{GaAs} \mathrm{emitter,} 30 \mathrm{~nm} \mathrm{p} \mathrm{Al}_{0.75} \mathrm{Ga}_{0.25} \mathrm{As}$ window layer, and $\mathrm{p}^{+} 50 \mathrm{~nm}$ GaAs contact layer. The intrinsic region of the SCs consists of 20 stacks of InAs QDs with $2 \mathrm{ML}$ AlAs CLs separated by a $20 \mathrm{~nm}$ GaAs spacer. The QDs were grown by the Stranski-Krastanov mode at $\sim 500{ }^{\circ} \mathrm{C}$. The QDSCs were Si-doped with four different doping densities of 0, 6, 12, and $18 \mathrm{e} / \mathrm{dot}$.

Atomic-force microscopy (AFM) was used to analyse the morphology of the InAs QDs grown on GaAs. The average diameter of the QDs measured from the AFM image (shown in

Figure 2) was $\sim 30 \mathrm{~nm}$, with an average height of $\sim 5 \mathrm{~nm}$. No large defective clusters were observed, which indicates a high structural quality ${ }^{18}$. The in-plane density of the InAs QDs was estimated to be $\sim 2.3 \times 10^{10} \mathrm{~cm}^{-2}$.

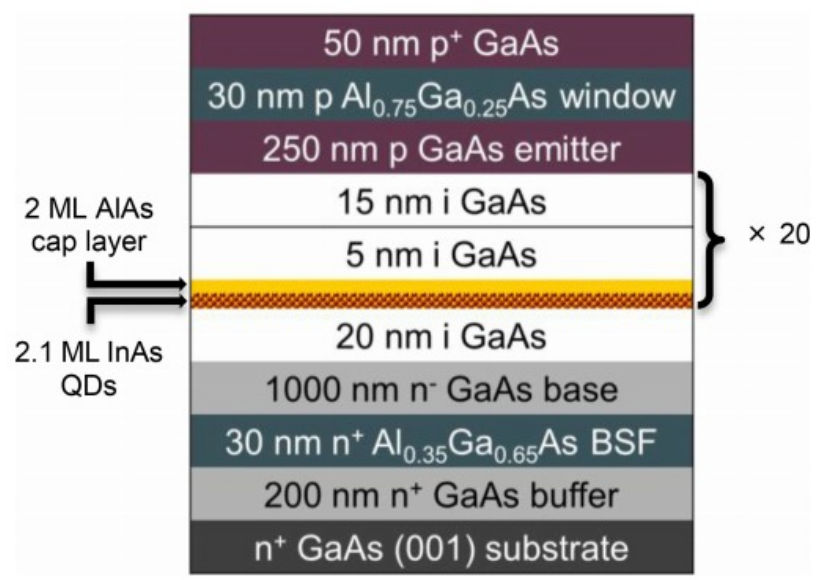

Figure 1 Structure of the Si-doped (0, 6, 12, 18 e/dot) InAs/GaAs QDSCs with AlAs CLs. 


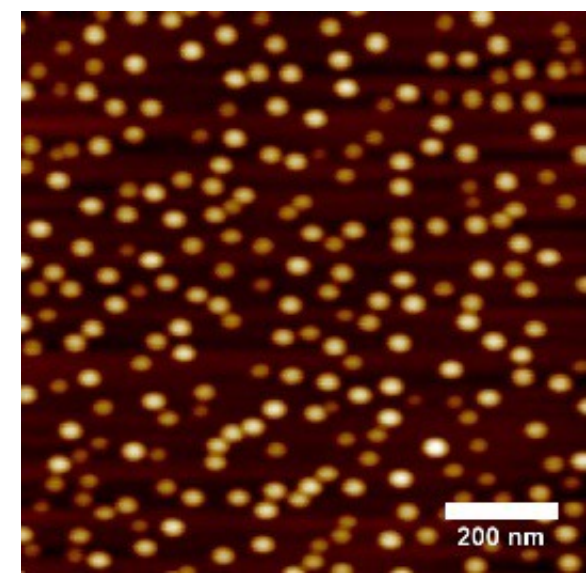

Figure 2 AFM images of InAs QDs grown on GaAs in 2D (top) and 3D (bottom).
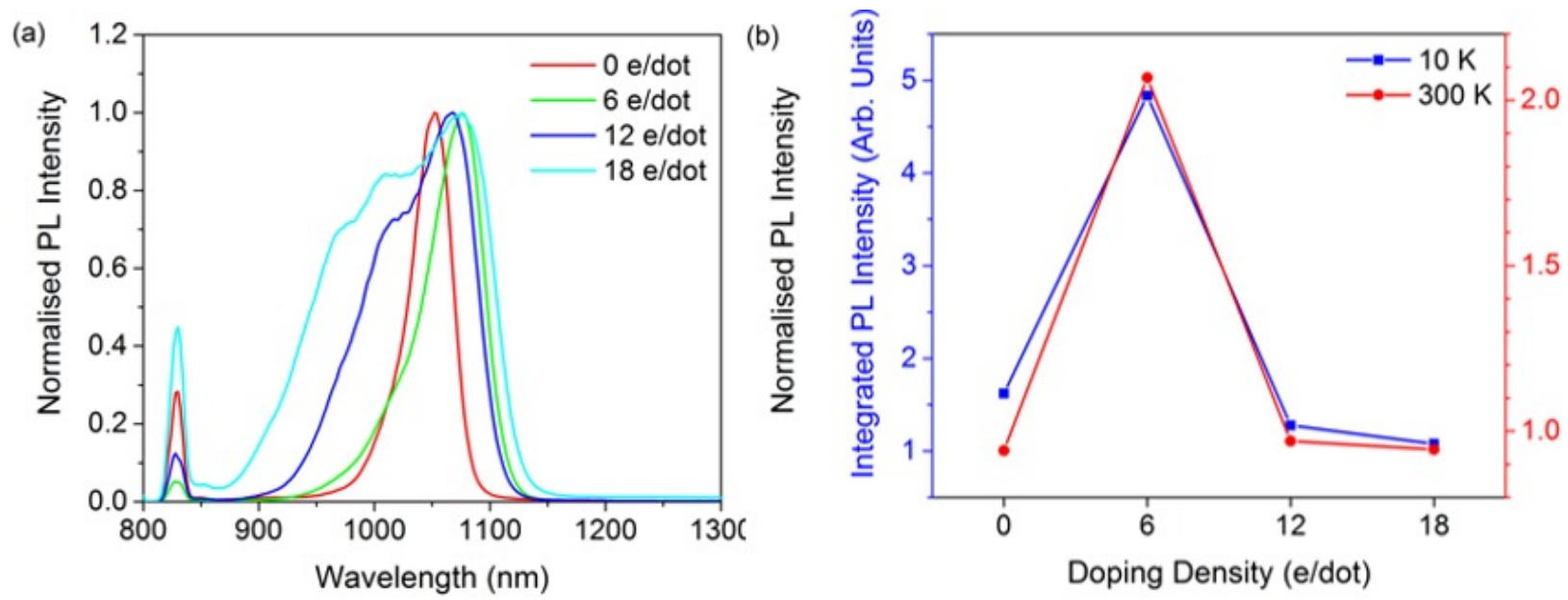

Figure 3 (a) Normalised PL spectra of Si-doped QDSCs with AlAs cap layers measured at $10 \mathrm{~K}\left(\mathrm{P}_{\mathrm{ex}}=37 \mathrm{~mW}\right)$, and (b) Integrated PL intensity vs. Si doping density at $10 \mathrm{~K}\left(\mathrm{I}_{\mathrm{ex}}=386 \mathrm{~W} / \mathrm{cm}^{2}, \lambda_{\mathrm{ex}}=532 \mathrm{~nm}\right)$ and $300 \mathrm{~K}\left(\mathrm{I}_{\mathrm{ex}}=459 \mathrm{~W} / \mathrm{cm}^{2}, \lambda_{\mathrm{ex}}=635\right.$ $\mathrm{nm})$.

\section{OPTICAL CHARACTERISTICS}

The optical properties of the Si-doped InAs/GaAs QDs with AlAs CLs were compared. Figure 3 (a) shows the normalised PL spectra for the QDSCs at $10 \mathrm{~K}$. The spectra display additional QD emission peaks $(950-1100 \mathrm{~nm})$ for the samples with higher Si doping. This can be explained by the emissions related to excited state transitions observed at shorter wavelengths alongside the ground state emission ${ }^{19}$. In contrast to our previous study ${ }^{9}$, the formation of WLs by applying AlAs CLs on QDs during the MBE growth ${ }^{8}$. In this manner, most Si dopants are concentrated inside the QDs rather than the WL, and contributes to state filling. Figure 3 (b) shows the integrated PL intensity as a function of Si doping density, at $10 \mathrm{~K}$ and $300 \mathrm{~K}$. It can be seen that the QDSC with doping density of 6 e/dot exhibits the strongest PL peak intensity. The enhancement of the PL intensity can be attributed to the passivation of the defect states by Si doping, which leads to suppression of the non-radiative process ${ }^{20,21}$. Figure 3 (b) shows that any further increase in the Si doping level results in a dramatic decrease in the PL intensity. This can be due the presence of too many Si atoms, which can cause destruction of the crystal lattice of the InAs QDs and formation of non-radiative recombination centres ${ }^{13}$. 
In order to provide further evidence of the QD state filling and the passivation of defect states achieved by Si doping, a time-resolved PL study was performed for the QDCSs at $10 \mathrm{~K}$. Figure 4 shows that the carrier lifetime in the region of $875-925 \mathrm{~nm}$ increases with increasing Si doping density. This can be linked to the state filling effect observed from the steady-state PL measurements in Figure 3 (a). The appearance of the higher-energy QD emission peaks with higher Si doping densities in Figure 3 (a) indicates that the additional electrons supplied by the Si dopants are used to fill the QD states. With more QD states filled with electrons, fewer available decay channels exist for the carriers, which in turn results in longer carrier lifetimes. In contrast, the carrier lifetime of the QD ground state $(1050-1100 \mathrm{~nm})$ in Figure 4 shows that increasing the Si doping density leads to an initial increase with moderate doping density (6 e/dot), followed by a significant drop in the carrier lifetime with higher doping densities $(12,18 \mathrm{e} / \mathrm{dot})$. This trend is similar to that observed in the integrated PL intensity measurements, as shown in Figure 3 (b). Therefore, it can be inferred that the initial increase in the carrier lifetime with doping density of 6 e/dot originates from the passivated defect states. However, once all defect states are filled, further addition of $\mathrm{Si}$ atoms leads to the formation of non-radiative recombination centres, and/or increase Auger scattering rate, which decreases the carrier lifetime.

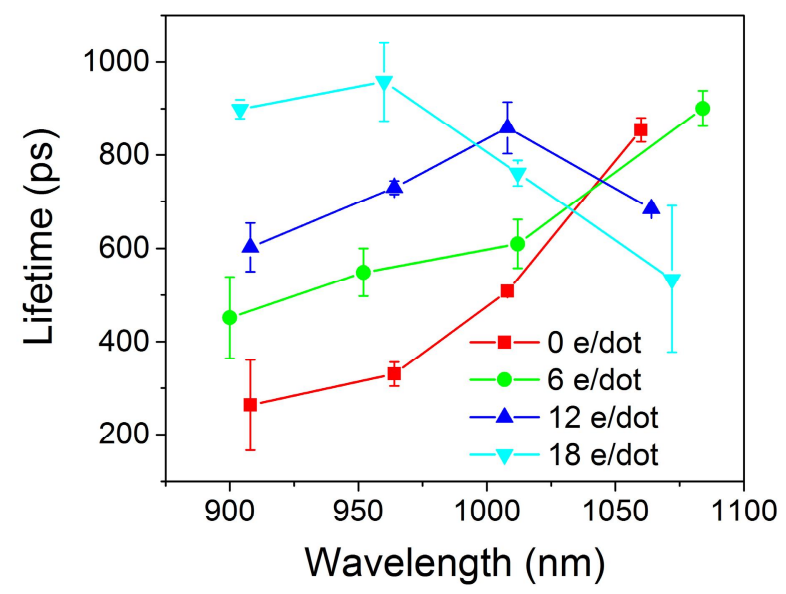

Figure 4 Carrier lifetime versus wavelength obtained from the transient photoluminescence spectra of the Si-doped QDSCs with AlAs cap layers at $10 \mathrm{~K}\left(\lambda_{\mathrm{ex}}=750 \mathrm{~nm}\right)$

\section{SOLAR CELL PERFORMANCES}

The EQE spectra of the Si-doped QDSCs with AlAs CLs are presented in Figure 5. For all doped samples, there is a drop in EQE at $\sim 870 \mathrm{~nm}$ which corresponds to the bandgap of GaAs. At the higher wavelengths, flat spectral responses without a WL peak $(\sim 915 \mathrm{~nm})$ are observed. It can be also noted from Figure 5 that the supra-bandgap $(400-870 \mathrm{~nm})$ absorption of all QDSCs with Si doping is significantly reduced when compared with that of the undoped QDSC. This can be attributed to the decrease in depletion region after Si doping, which reduces the effective absorption area.

The J-V characteristics of the QDSCs in Figure 6 show a clear dependence on the Si-doping density. Firstly, all Si-doped samples display a significant drop in the current density when compared with the undoped sample. This can be related to the reduction in the EQE contribution from the supra-bandgap region observed in Figure 5. Secondly, a further gradual decrease in the current density with increasing doping is observed amongst the doped QDSCs. This is likely to be due to the point defects formed by Si dopants substituting Ga and As or existing as interstitials ${ }^{22,23}$. Although Si doping has a negative impact in the current density, an enhancement of the $\mathrm{V}_{\mathrm{OC}}$ is observed with moderate doping density, which is likely to be due to the moderate Si doping passivating the defect states. 


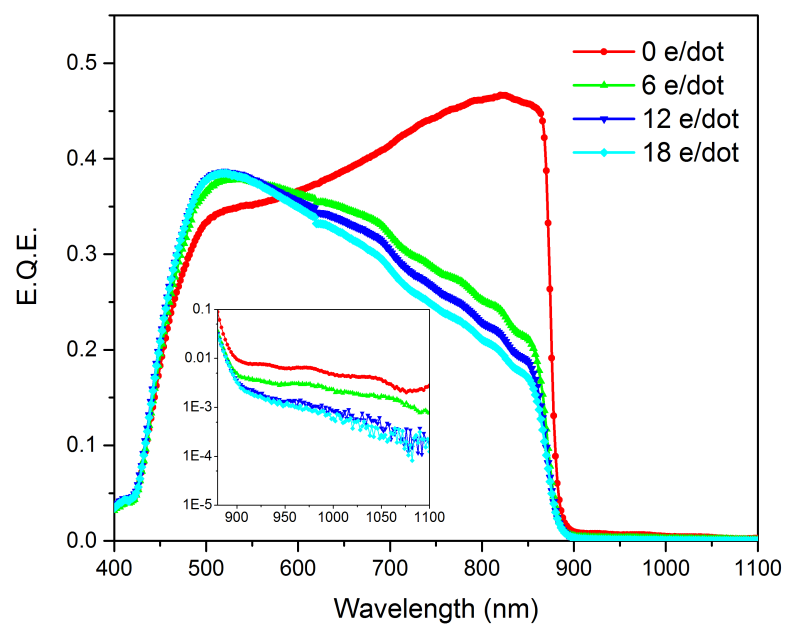

Figure 5 Linear-scale external quantum efficiency spectra of Si-doped QDSCs with AlAs cap layers. Inset shows semi-log scale of sub-bandgap external quantum efficiency.

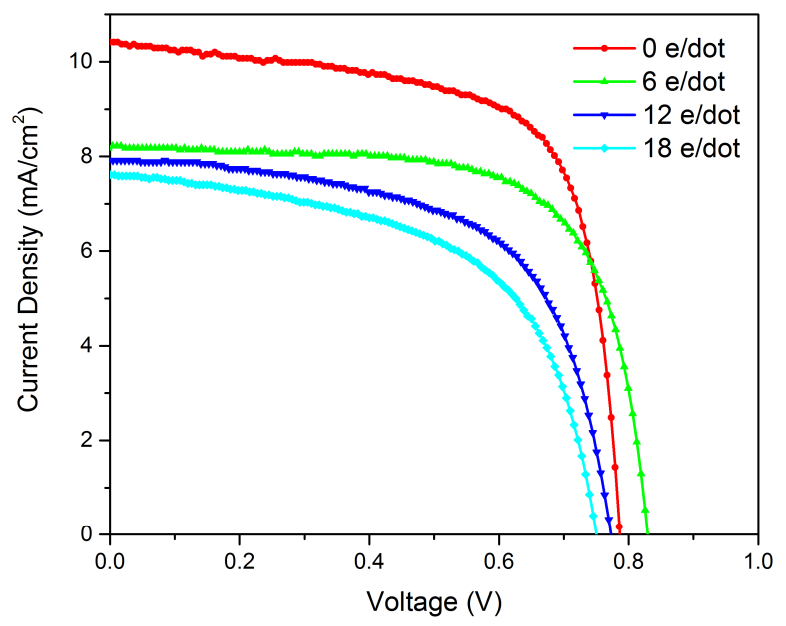

Figure 6 Current density versus voltage behaviour of Si-doped QDSCs with AlAs cap layers under one-sun (AM .5G) illumination.

\section{CONCLUSION}

In conclusion, an improvement in the $\mathrm{V}_{\mathrm{OC}}(\sim 44 \mathrm{mV})$ has been achieved by introducing direct Si doping to QDs with AlAs CLs. The increase in the $\mathrm{V}_{\mathrm{OC}}$ is attributed to the passivation of the defect states with moderate Si doping (6 e/dot), alongside the reduced CB-IB thermal coupling with AlAs CLs. Also, with the AlAs CLs, the QD state filling effect is observed with low Si doping densities. The factors that contributed to the decrease in the supra-bandgap EQE and the photocurrent after Si doping, such as the decrease in depletion region and the formation of point defects, should be taken into account when designing future Si-doped QDSCs. Nonetheless, the results presented in this article hold some promise for overcoming some of the main challenges in implementing QD-IBSCs with the efficiencies close to that of the theoretical model of the IBSC. 


\section{REFERENCES}

[1] Shockley, W., and Queisser, H.J., "Detailed Balance Limit of Efficiency of p n Junction Solar Cells," Journal of Applied Physics 32(3), 510-519 (1961).

[2] Luque, A., Martí, A., and Stanley, C., "Understanding intermediate-band solar cells," Nature Photonics 6(3), 146-152 (2012).

[3] Sanguinetti, S., Henini, M., Grassi Alessi, M., Capizzi, M., Frigeri, P., and Franchi, S., "Carrier thermal escape and retrapping in self-assembled quantum dots," Physical Review B 60(11), 8276-8283 (1999).

[4] Antolín, E., Martí, A., Farmer, C.D., Linares, P.G., Hernández, E., Sánchez, A.M., Ben, T., Molina, S.I., Stanley, C.R., et al., "Reducing carrier escape in the InAs/GaAs quantum dot intermediate band solar cell," Journal of Applied Physics 108(6), 64513 (2010).

[5] Luque, A., and Martí, A., "The Intermediate Band Solar Cell: Progress Toward the Realization of an Attractive Concept," Advanced Materials 22(2), 160-174 (2010).

[6] Bailey, C.G., Forbes, D.V., Raffaelle, R.P., and Hubbard, S.M., "Near 1 V open circuit voltage InAs/GaAs quantum dot solar cells," Applied Physics Letters 98(16), 163105 (2011).

[7] Mellor, A., Luque, A., Tobías, I., and Martí, A., "Realistic Detailed Balance Study of the Quantum Efficiency of Quantum Dot Solar Cells," Advanced Functional Materials 24(3), 339-345 (2014).

[8] Tutu, F.K., Lam, P., Wu, J., Miyashita, N., Okada, Y., Lee, K.-H., Ekins-Daukes, N.J., Wilson, J., and Liu, H., "InAs/GaAs quantum dot solar cell with an AlAs cap layer," Applied Physics Letters 102(16), 163907 (2013).

[9] Lam, P., Hatch, S., Wu, J., Tang, M., Dorogan, V.G., Mazur, Y.I., Salamo, G.J., Ramiro, I., Seeds, A., et al., "Voltage recovery in charged InAs/GaAs quantum dot solar cells," Nano Energy 6, 159-166 (2014).

[10] Liu, H.Y., Sellers, I.R., Badcock, T.J., Mowbray, D.J., Skolnick, M.S., Groom, K.M., Gutiérrez, M., Hopkinson, M., Ng, J.S., et al., "Improved performance of $1.3 \mu \mathrm{m}$ multilayer InAs quantum-dot lasers using a high-growthtemperature GaAs spacer layer," Applied Physics Letters 85(5), 704-706 (2004).

[11] Tutu, F.K., Sellers, I.R., Peinado, M.G., Pastore, C.E., Willis, S.M., Watt, A.R., Wang, T., and Liu, H.Y., "Improved performance of multilayer InAs/GaAs quantum-dot solar cells using a high-growth-temperature GaAs spacer layer," Journal of Applied Physics 111(4), 46101 (2012).

[12] Martí, A., López, N., Antolín, E., Cánovas, E., Luque, A., Stanley, C.R., Farmer, C.D., and Díaz, P., "Emitter degradation in quantum dot intermediate band solar cells," Applied Physics Letters 90(23), 233510 (2007).

[13] Yang, X., Wang, K., Gu, Y., Ni, H., Wang, X., Yang, T., and Wang, Z., "Improved efficiency of InAs/GaAs quantum dots solar cells by Si-doping," Solar Energy Materials and Solar Cells 113, 144-147 (2013).

[14] Sugaya, T., Kamikawa, Y., Furue, S., Amano, T., Mori, M., and Niki, S., "Multi-stacked quantum dot solar cells fabricated by intermittent deposition of InGaAs," Solar Energy Materials and Solar Cells 95(1), 163-166 (2011).

[15] Martí, A., Cuadra, L., and Luque, A., "Partial filling of a quantum dot intermediate band for solar cells," IEEE Transactions on Electron Devices 48(10), 2394-2399 (2001).

[16] Martí, A., López, N., Antolín, E., Cánovas, E., Stanley, C., Farmer, C., Cuadra, L., and Luque, A., "Novel semiconductor solar cell structures: The quantum dot intermediate band solar cell," Thin Solid Films 511-512, 638-644 (2006).

[17] Luque, A., and Martí, A., "A metallic intermediate band high efficiency solar cell," Progress in Photovoltaics: Research and Applications 9(2), 73-86 (2001).

[18] Liu, H.Y., Sellers, I.R., Gutiérrez, M., Groom, K.M., Soong, W.M., Hopkinson, M., David, J.P.R., Beanland, R., Badcock, T.J., et al., "Influences of the spacer layer growth temperature on multilayer InAs/GaAs quantum dot structures," Journal of Applied Physics 96(4), 1988-1992 (2004).

[19] Marquezini, M.V., Brasil, M.J.S.P., Brum, J.A., Poole, P., Charbonneau, S., and Tamargo, M.C., "Study of temperature-dependent exciton dynamics in a single quantum well with self-assembled islands," Surface Science 361-362, 810-813 (1996).

[20] Sablon, K.A., Little, J.W., Mitin, V., Sergeev, A., Vagidov, N., and Reinhardt, K., "Strong Enhancement of Solar Cell Efficiency Due to Quantum Dots with Built-In Charge," Nano Letters 11(6), 2311-2317 (2011).

[21] Inoue, T., Kido, S., Sasayama, K., Kita, T., and Wada, O., "Impurity doping in self-assembled InAs/GaAs quantum dots by selection of growth steps," Journal of Applied Physics 108(6), 63524 (2010).

[22] Hurle, D.T.J., "A comprehensive thermodynamic analysis of native point defect and dopant solubilities in gallium arsenide," Journal of Applied Physics 85(10), 6957-7022 (1999).

[23] Hurle, D.T.J., "A thermodynamic analysis of native point defect and dopant solubilities in zinc-blende III-V semiconductors," Journal of Applied Physics 107(12), 121301 (2010). 